\title{
Dynamic Multi-threshold Metering Schemes
}

\author{
Carlo Blundo ${ }^{1}$, Annalisa De Bonis ${ }^{1}$, Barbara Masucci ${ }^{1}$, and \\ Douglas R. Stinson ${ }^{2}$ \\ 1 Dipartimento di Informatica ed Applicazioni, Università di Salerno, \\ 84081 Baronissi (SA), Italy, \\ \{carblu, debonis, masucci\}@dia.unisa.it \\ http://www.dia.unisa.it/ $\{$ carblu, masucci\} \\ 2 Department of Combinatorics and Optimization, University of Waterloo, \\ Waterloo, Ontario, N2L 3G1, Canada, \\ dstinson@cacr.math. uwaterloo.ca \\ http://www.cacr.math.uwaterloo.ca/ ${ }^{\sim}$ dstinson
}

\begin{abstract}
A metering scheme is a protocol in which an audit agency is able to measure the interaction between clients and servers on the web during a certain number of time frames. Naor and Pinkas [7] considered metering schemes in which any server is able to construct a proof to be sent to the audit agency if and only if it has been visited by at least a number, say $h$, of clients in a given time frame. In their schemes the parameter $h$ is fixed and is the same for any server and any time frame. In this paper we introduce dynamic multi-threshold metering schemes, that are metering schemes in which there is a threshold associated to any server for any time frame. We mainly focus on the efficiency of dynamic multi-threshold metering schemes, by minimizing the information received and distributed by clients. This is important because the clients participating in the metering process do not receive any money from the audit agency.
\end{abstract}

Keywords: Metering Schemes, Security, Cryptography, Entropy.

\section{Introduction}

Most of the revenues of web sites come from advertisement payments. Web advertisers must have a way to measure the exposure of their ads by obtaining usage statistics about web sites which contain their ads. Indeed, the amount of money charged to display ads depends on the number of visits received by the web site. Consequently, advertisers should prevent the web sites from inflating the count of their visits in order to demand more money. Hence, there should be a mechanism which ensures the validity and accuracy of usage measurements against fraud attempts by servers (web sites) and clients (visitors). In a typical scenario there are many servers and clients, and an audit agency whose task is to measure the interaction between the servers and the clients. A system for measuring the amount of services performed by the servers is called metering scheme.

D.R. Stinson and S. Tavares (Eds.): SAC 2000, LNCS 2012, pp. 130-144 2001.

(C) Springer-Verlag Berlin Heidelberg 2001 
Naor and Pinkas 7] proposed metering schemes in which any server is able to present to the audit agency a short proof for the number of client visits it has received in a given time frame. In their schemes all servers are associated to a threshold $h$, and are able to compute their proofs for a certain time frame if and only if they have been visited by a number of clients larger than or equal to $h$ in that time frame. The schemes proposed by Naor and Pinkas are also efficient: the task for the audit agency in sending information to clients and servers is very simple, as well as the task for the servers in computing their proofs. Recently, different kinds of metering schemes have been proposed. Metering schemes for ramp structures [13] have been introduced in order to reduce the overhead to the overall communication due to the metering process. Metering schemes with pricing [1,5] have been introduced in order to have a more flexible payment system. Finally, metering schemes for general access structures [6] have been introduced in order to measure the interaction between servers and particular groups of clients.

In metering schemes considered by Naor and Pinkas [7] the parameter $h$ is fixed and is the same for any server and any time frame. This is acceptable whenever there is a long-term relationship between the audit agency and the servers. In order to measure any number of visits in any granularity we introduce dynamic multi-threshold metering schemes, which are metering schemes in which there is a threshold $h_{j}^{t}$ associated to any server $\mathcal{S}_{j}$ for any time frame $t$.

Dynamic multi-threshold metering schemes involve distributing information to clients and servers. Obviously, such information distribution affects the overall communication complexity. Therefore, it is important to construct schemes whose overhead to the overall communication is as small as possible. We mainly focus on the efficiency of dynamic multi-threshold metering schemes, by minimizing the information received and distributed by clients. This is important because the clients participating in the metering process do not receive any money from the audit agency. In this paper we provide lower bounds on the size of the information received and distributed by clients and we present a scheme achieving these lower bounds.

\section{The Model}

Consider the following scenario: there are $n$ clients, $m$ servers and an audit agency $A$ which is interested in counting the client visits to the servers in $\tau$ different time frames. For any $i=1, \ldots, n$ and $j=1, \ldots, m$, we denote by $\mathcal{C}_{i}$ the $i$-th client and by $\mathcal{S}_{j}$ the $j$-th server.

There is an initialization phase in which the audit agency $A$ distributes some information to any client over a private channel. For any $i=1, \ldots, n$, we denote by $c_{i}$ the information that the audit agency $A$ gives to the client $\mathcal{C}_{i}$. Moreover, we denote by $C_{i}$ the set of all values that $c_{i}$ can assume. Given a set of client indices $Z=\{1, \ldots, \alpha\} \subseteq\{1, \ldots, n\}$, we denote by $C_{z}$ the cartesian product $C_{1} \times \cdots \times C_{\alpha}$. 
At the beginning of any time frame the audit agency $A$ distributes to any server a piece of information which depends on the identity of the server and on the time frame. For any $j=1, \ldots, m$ and $t=1, \ldots, \tau$, we denote by $s_{j}^{t}$ the information that the audit agency $A$ gives to the server $\mathcal{S}_{j}$ at the beginning of time frame $t$. Moreover, we denote by $S_{j}^{t}$ the set of all values that $s_{j}^{t}$ can assume. Given a set of server indices $B=\{1, \ldots, \beta\} \subseteq\{1, \ldots, m\}$, we denote by $S_{B}^{t}$ the cartesian product $S_{1}^{t} \times \cdots \times S_{\beta}^{t}$.

A regular operation consists in a client visit to a server during a time frame. During such a visit the client gives to the visited server a piece of information which depends on its private information, on the identity of the server, and on the time frame during which the client visits the server. For any $i=1, \ldots, n$, $j=1, \ldots, m$, and $t=1, \ldots, \tau$, we denote by $c_{i, j}^{t}$ the information that the client $\mathcal{C}_{i}$ sends to the server $\mathcal{S}_{j}$ when visiting it in time frame $t$. Moreover, we denote by $C_{i, j}^{t}$ the set of all values that $c_{i, j}^{t}$ can assume. Given a set of server indices $B=$ $\{1, \ldots, \beta\} \subseteq\{1, \ldots, m\}$, we denote by $C_{i, B}^{t}$ the cartesian product $C_{i, 1}^{t} \times \cdots \times C_{i, \beta}^{t}$. Moreover, given a set of client indices $Z=\{1, \ldots, \alpha\} \subseteq\{1, \ldots, n\}$, we denote by $C_{Z, B}^{t}$ the cartesian product $C_{1, B}^{t} \times \cdots \times C_{\alpha, B}^{t}$. For any $j=1, \ldots, m$ and $t=1, \ldots, \tau$, we denote by $X_{j,\left(d_{j}\right)}^{t}$ the set of the $d_{j}$ client visits received by server $\mathcal{S}_{j}$ in time frame $t$.

During the proof computation stage any server $\mathcal{S}_{j}$ which has received at least $h_{j}^{t}$ visits during time frame $t$ is able to compute its proof for time frame $t$, as function of the information provided by the $h_{j}^{t}$ clients and the information $s_{j}^{t}$ provided by the audit agency $A$ at the beginning of the time frame $t$. For any $j=1, \ldots, m$ and $t=1, \ldots, \tau$, we denote by $p_{j}^{t}$ the proof computed by the server $\mathcal{S}_{j}$ when it has been visited by at least $h_{j}^{t}$ distinct clients in time frame $t$. Moreover, we denote by $P_{j}^{t}$ the set of all values that $p_{j}^{t}$ can assume. Given a set of server indices $B=\{1, \ldots, \beta\} \subseteq\{1, \ldots, m\}$, we denote by $P_{B}^{t}$ the cartesian product $P_{1}^{t} \times \cdots \times P_{\beta}^{t}$.

During the proof verification stage the audit agency $A$ verifies the proofs received by servers and decides on the amount of money to be paid to servers. If the proof received from a server at the end of a time frame is correct, then $A$ pays the server for its services.

A corrupt server can be assisted by corrupt clients and other corrupt servers in order to inflate the count of its visits. A corrupt client $\mathcal{C}_{i}$ can donate to a corrupt server the whole private information received by the audit agency during the initialization phase. We assume that the number of corrupt clients is $c$, where $1 \leq c<\min _{j=1, \ldots, m} \min _{t=1, \ldots, \tau} h_{j}^{t}$. A corrupt server can donate to another corrupt server the private information received from the audit agency at the beginning of any time frame in addition to the information received from clients in previous time frames and in the actual time frame. For any $i=1, \ldots, n$ and $t=1, \ldots, \tau$, we denote by $V_{j}^{[t]}$ all the information received by a corrupt server $\mathcal{S}_{j}$ in time frames $1, \ldots, t$. This information includes the sets of client visits received by server $\mathcal{S}_{j}$ in time frames $1, \ldots, t$. We also define $V_{j}^{[0]}=\emptyset$, for any corrupt server $\mathcal{S}_{j}$. We assume that the maximum number of corrupt servers 
is $s$, where $1 \leq s \leq m$. For the reader's convenience, the notations used in this section are summarized in Appendix B.

In this paper with a boldface capital letter, say $\mathbf{X}$, we denote a random variable taking value on a set denoted by the corresponding capital letter $X$ according to some probability distribution $\left\{\operatorname{Pr}_{\mathbf{X}}(x)\right\}_{x \in X}$. The values such a random variable can take are denoted by the corresponding lower letter. Given a random variable $\mathbf{X}$ we denote with $H(\mathbf{X})$ the Shannon entropy of $\left\{\operatorname{Pr}_{\mathbf{X}}(x)\right\}_{x \in X}$ (for some basic properties of entropy, consult the Appendix A).

We formally define dynamic multi-threshold metering schemes by using the entropy approach, as done in $[1,3,5 ! 6]$. We use the entropy approach mainly because this leads to a compact and simple description of the schemes and because the entropy approach takes into account all probability distributions on the sets of the proofs computed by the servers.

Definition 1. An $\left(n, m, \tau, c, s,\left\{h_{j}\right\}_{j=1, \ldots, m}^{t=1, \ldots, \tau}\right)$ dynamic multi-threshold metering scheme is a protocol to measure the interaction between $n$ clients and $m$ servers during $\tau$ time frames in such a way that the following properties are satisfied:

1. Any client is able to compute the information needed to visit any server in any time frame:

Formally, it holds that $H\left(\mathbf{C}_{i, j}^{t} \mid \mathbf{C}_{i}\right)=0$ for $i=1, \ldots, n, j=1, \ldots, m$, and $t=1, \ldots, \tau$.

2. Any server $\mathcal{S}_{j}$ which has received $h_{j}^{t}$ client visits during time frame $t$ and the message provided by $A$ at the beginning of the time frame $t$ can compute its proof for $t$ :

Formally, it holds that $H\left(\mathbf{P}_{j}^{t} \mid \mathbf{X}_{j,\left(h_{j}^{t}\right)}^{t} \mathbf{S}_{j}^{t}\right)=0$, for $j=1, \ldots, m$ and $t=$ $1, \ldots, \tau$.

3. Let us consider a coalition of $\alpha$ corrupt clients $\mathcal{C}_{1}, \ldots, \mathcal{C}_{\alpha}$ and $\beta$ corrupt servers $\mathcal{S}_{1}, \ldots, \mathcal{S}_{\beta}$, where $0 \leq \alpha \leq c<\min _{j=1, \ldots, m} \min _{t=1, \ldots, \tau} h_{j}^{t}$ and $1 \leq$ $\beta \leq s$, and let $B=\{1, \ldots, \beta\}$. Assume that at some time frame $t$ each server $\mathcal{S}_{j}$ in the coalition has been visited by less than $h_{j}^{t}-\alpha$ clients and has received the information by $A$. Then, the servers in the coalition have no information on their proofs for $t$ :

Formally, it holds that $H\left(\mathbf{P}_{B}^{t} \mid \mathbf{C}_{1} \ldots \mathbf{C}_{\alpha} \mathbf{X}_{1,\left(d_{1}\right)}^{t} \ldots \mathbf{X}_{\beta,\left(d_{\beta}\right)}^{t} \mathbf{S}_{B}^{t} \mathbf{V}_{B}^{[t-1]}\right)=$ $H\left(\mathbf{P}_{B}^{t}\right)$, where $d_{j}<h_{j}^{t}-\alpha$, for $j=1, \ldots, \beta$.

Notice that Naor and Pinkas [7] considered metering schemes which are "static" and with "single threshold", i.e., where $h_{j}^{t}=h$ for $j=1, \ldots, m$ and $t=1, \ldots, \tau$. Moreover, their schemes do not require communication between audit agency and servers at the beginning of any time frame.

\section{A Dynamic Multi-threshold Metering Protocol}

In this section we present a dynamic multi-threshold metering scheme which is optimal with respect to the bounds (4) and (5) presented in Section 4. The protocol is a generalization of Naor and Pinkas metering scheme [7]. 
Initialization: For $j=1, \ldots, m$ and $t=1, \ldots, \tau$, let $h_{j}^{t}$ be the threshold associated to the server $\mathcal{S}_{j}$ in time frame $t$ and let $h=\max _{j=1, \ldots, m} \max _{t=1, \ldots, \tau} h_{j}^{t}+1$. The audit agency $A$ chooses a random polynomial $Q(x, y)$ of degree $h-1$ in $x$ and $s \tau-1$ in $y$ over $G F(q)$, where $q$ is a sufficiently large prime number. Afterwards, $A$ sends the univariate polynomial $Q(i, y)$, which is of degree $s \tau-1$, to each client $\mathcal{C}_{i}$.

Beginning of a Time Frame: At the beginning of time frame $t$, for any server $\mathcal{S}_{j}$, the audit agency $A$ evaluates the polynomial $Q(x, j \circ t)$ in $h-h_{j}^{t}$ points other than $1, \ldots, n$ and sends these values to $\mathcal{S}_{j}$. The argument $j \circ t$ denotes the concatenation of $j$ and $t$, and we assume for simplicity that $j \circ t$ is in $G F(q)$ and that no distinct two pairs $(j, t)$ and $\left(j^{\prime}, t^{\prime}\right)$ are mapped to the same element.

Regular Operation: When the client $\mathcal{C}_{i}$ visits the server $\mathcal{S}_{j}$ in time frame $t$, it sends the value $Q(i, j \circ t)$ to $\mathcal{S}_{j}$.

Proof Generation and Verification: Assume that the server $\mathcal{S}_{j}$ has been visited by at least $h_{j}^{t}$ different clients in time frame $t$. Then, knowing the $h-h_{j}^{t}$ points of $Q(x, j \circ t)$ provided by the audit agency at the beginning of time frame $t$, the server can perform a Lagrange interpolation and reconstruct the polynomial $Q(x, j \circ t)$. Then, it can compute the value $Q(0, j \circ t)$, which constitutes the proof that the server sends to the audit agency. The audit agency can easily verify this value.

\subsection{Security of the Scheme}

In this section we prove that the scheme presented in Section 3 satisfies Properties 1, 2, and 3 of Definition 1

It is immediate to verify that the scheme satisfies Property 1 of Definition 1. Indeed, for any $i=1, \ldots, n$, the information given by the audit agency to the client $\mathcal{C}_{i}$ consists of the univariate polynomial $Q(i, y)$ and for any $j=1, \ldots, m$ and $t=1, \ldots, \tau$, the information given to the server $\mathcal{S}_{j}$ by client $\mathcal{C}_{i}$ in time frame $t$ is obtained by evaluating the univariate polynomial $Q(i, y)$ at $j \circ t$.

It is also easy to verify that the scheme satisfies Property 2 of Definition 1 Assume that a server $\mathcal{S}_{j}$ has been visited by $h_{j}^{t}$ clients in time frame $t$ and that it has received $h-h_{j}^{t}$ points of $Q(x, j \circ t)$ from the audit agency at the beginning of time frame $t$. Therefore, the server $\mathcal{S}_{j}$ knows $h$ points of the polynomial $Q(x, j \circ t)$ and can perform a Lagrange interpolation on it. Afterwards, it can compute its proof $Q(0, j \circ t)$ by evaluating the polynomial $Q(x, j \circ t)$ at the point 0 .

Finally, we prove that the scheme satisfies Property 3 of Definition $10 \mathrm{We}$ consider the worst possible case in which $c$ corrupt clients decide to cooperate with $s$ corrupt servers at time frame $\tau$. Moreover we assume that the corrupt servers have collected the maximum possible information during the previous time frames $1, \ldots, \tau-1$. In other words, we assume that each corrupt client $\mathcal{C}_{i}$ 
gives its polynomial $Q(i, y)$ to all servers in the coalition, and that any corrupt server $\mathcal{S}_{j}$ in the coalition knows the polynomial $Q(x, j \circ t)$ for $t=1, \ldots, \tau-1$.

In order to compute its proof $Q(0, j \circ \tau)$ for time frame $\tau$, any server $\mathcal{S}_{j}$ should be able to interpolate either the polynomial $Q(x, j \circ \tau)$ or the bivariate polynomial $Q(x, y)$. Notice that for any $j, k \in\{1, \ldots, s\}$, with $j \neq k$, the information held by the server $\mathcal{S}_{k}$ is of no help in computing the polynomial $Q(x, j \circ \tau)$. Assume $g_{j}=h_{j}^{\tau}-c-1$ be the number of client visits received by server $\mathcal{S}_{j}$ during time frame $\tau$. Each corrupt client $\mathcal{C}_{i}$ donates to $\mathcal{S}_{j}$ the polynomial $Q(i, y)$ from which $\mathcal{S}_{j}$ can compute the value $Q(i, j \circ \tau)$. Since there are $c$ corrupt clients, $\mathcal{S}_{j}$ can compute $c$ values of $Q(x, j \circ \tau)$ in addition to those provided by the $g_{j}$ visits performed by non corrupt clients. Since the server $\mathcal{S}_{j}$ has also received $h-h_{j}^{\tau}$ points of $Q(x, j \circ \tau)$ by the audit agency at the beginning of time frame $\tau$, the overall number of points of $Q(x, j \circ \tau)$ known to $\mathcal{S}_{j}$ is $g_{j}+c+h-h_{j}^{\tau}=h-1$. Therefore, the server obtains a linear system of $h-1$ equations in $h$ unknowns. For any choice of a value in $G F(q)$, there is a polynomial $R(x, j \circ \tau)$ which is consistent with the information held by the server. Since there are $q$ such polynomials, the probability of the server in guessing its proof for time frame $\tau$ is at most $1 / q$.

Alternatively, the coalition of corrupt servers might try to interpolate the polynomial $Q(x, y)$ in order to compute the proofs. The information that a corrupt client $\mathcal{C}_{i}$ gives to a corrupt server is equivalent to the $s \tau$ coefficients of its polynomial $Q(i, y)$. For $j=1, \ldots, s$, the information collected by each corrupt server $\mathcal{S}_{j}$ at the beginning of time frame $\tau$ is constituted by the information provided by the audit agency at the beginning of any time frame $t=1, \ldots, \tau$, which consists in $h-h_{j}^{t}$ coefficients of $Q(x, j \circ t)$, in addition to the information provided by clients during each time frame $t=1, \ldots, \tau-1$, which consists in $h_{j}^{t}$ coefficients of $Q(x, j \circ t)$. Hence, at the beginning of time frame $\tau$ each corrupt server holds $(\tau-1) h$ coefficients of $Q(x, y)$ and $h-h_{j}^{\tau}$ coefficients of $Q(x, j \circ \tau)$. Suppose that in time frame $\tau$ each server $\mathcal{S}_{j}, j \in\{1, \ldots, s\}$, receives $g_{j} \leq h_{j}^{\tau}-\alpha-1$ regular visits from clients. Then, the overall information on $Q(x, y)$ held by the coalition of corrupt servers and clients at the end of time frame $\tau$ consists of

$$
c s \tau+s(\tau-1) h+\sum_{j=1}^{s}\left(h-h_{j}^{\tau}\right)+\sum_{j=1}^{s} g_{j}-c s(\tau-1)
$$

points. The first term of (11) corresponds to the information donated by the $c$ corrupt clients, the second term corresponds to the information collected by the $s$ corrupt servers during time frames $1, \ldots, \tau-1$, the third term corresponds to the information provided by the audit agency at the beginning of time frame $\tau$, the fourth term corresponds to the information provided by client visits at time frame $\tau$, and the last term corresponds to the information which has been counted twice. Since $g_{j} \leq h_{j}^{\tau}-\alpha-1$ for $j=1, \ldots, s$, it is easy to see that expression (1) is less than or equal to $h s \tau-s$. Therefore, the servers obtain a system of at most $h s \tau-s$ equations in $h s \tau$ unknowns. For any choice of $s$ values in $G F(q)$, there is a polynomial $R(x, y)$ which is consistent with the information 
held by the servers in the coalition. Since there are $q^{s}$ such polynomials, then the corrupt servers $\mathcal{S}_{1}, \ldots, \mathcal{S}_{s}$ have probability at most $1 / q^{s}$ of guessing their proofs for time frame $\tau$.

\section{Lower Bounds on the Size of the Information Distributed to Clients and Servers}

Dynamic multi-threshold metering schemes involve distributing information to clients and servers. In this section we provide lower bounds on the size of the information received by clients from the audit agency and distributed by clients to servers in dynamic multi-threshold metering schemes.

In order to prove our results we will resort to the two following technical lemmas.

Lemma 2. Let $\mathbf{A}$ and $\mathbf{E}$ be two random variables such that $H(\mathbf{A} \mid \mathbf{E})=0$. Then, for any two random variables $\mathbf{F}$ and $\mathbf{G}$, it holds that

$$
H(\mathbf{G} \mid \mathbf{A E F})=H(\mathbf{G} \mid \mathbf{E F})
$$

Proof. Consider the mutual information $I(\mathbf{A} ; \mathbf{G} \mid \mathbf{E F})$. From (12) of Appendix A it holds that

$$
H(\mathbf{A} \mid \mathbf{E F})-H(\mathbf{A} \mid \mathbf{E F G})=H(\mathbf{G} \mid \mathbf{E F})-H(\mathbf{G} \mid \mathbf{A E F})
$$

From (13) of Appendix A we have that $H(\mathbf{A} \mid \mathbf{E F G}) \leq H(\mathbf{A} \mid \mathbf{E F}) \leq H(\mathbf{A} \mid \mathbf{E})$. Since $H(\mathbf{A} \mid \mathbf{E})=0$, it follows that

$$
H(\mathbf{G} \mid \mathbf{A E F})=H(\mathbf{G} \mid \mathbf{E F}) .
$$

Lemma 3. Let $\mathbf{E}, \mathbf{F}$, and $\mathbf{G}$ be three random variables such that $H(\mathbf{G} \mid \mathbf{E F})=0$ and $H(\mathbf{G} \mid \mathbf{E})=H(\mathbf{G})$. Then, it holds that

$$
H(\mathbf{F} \mid \mathbf{E})=H(\mathbf{G})+H(\mathbf{F} \mid \mathbf{E G}) .
$$

Proof. Consider the mutual information $I(\mathbf{F} ; \mathbf{G} \mid \mathbf{E})$. From (12) of Appendix A it holds that

$$
H(\mathbf{F} \mid \mathbf{E})-H(\mathbf{F} \mid \mathbf{E G})=H(\mathbf{G} \mid \mathbf{E})-H(\mathbf{G} \mid \mathbf{E F}) .
$$

Since $H(\mathbf{G} \mid \mathbf{E F})=0$ and $H(\mathbf{G} \mid \mathbf{E})=H(\mathbf{G})$, then it follows that $H(\mathbf{F} \mid \mathbf{E})=$ $H(\mathbf{G})+H(\mathbf{F} \mid \mathbf{E G})$.

The next lemma immediately follows from Definition 1, Recall that for any sets of client and server indices $Z=\{1, \ldots, \alpha\} \subseteq\{1, \ldots, n\}$ and $B=\{1, \ldots, \beta\} \subseteq$ $\{1, \ldots, m\}$, respectively, we denote by $c_{z, B}^{t}$ the information given by clients $\mathcal{C}_{1}, \ldots, \mathcal{C}_{\alpha}$ to servers $\mathcal{S}_{1}, \ldots, \mathcal{S}_{\beta}$ during their visits in time frame $t$. 
Lemma 4. Let $\mathcal{M}$ be an $\left(n, m, \tau, c, s,\left\{h_{j}\right\}_{j=1, \ldots, m}^{t=1, \ldots, \tau}\right)$ dynamic multi-threshold metering scheme. Let $Z=\{1, \ldots, \alpha\}$ be a set of client indices and let $B=$ $\{1, \ldots, \beta\}$ be a set of server indices. Then, for any time frame $t=1, \ldots, \tau$, it holds that

$$
H\left(\mathbf{C}_{Z, B}^{t} \mid \mathbf{C}_{Z}\right)=0
$$

Proof. We have that

$$
\begin{aligned}
H\left(\mathbf{C}_{z, B}^{t} \mid \mathbf{C}_{Z}\right) & \leq \sum_{i=1}^{\alpha} \sum_{j=1}^{\beta} H\left(\mathbf{C}_{i, j}^{t} \mid \mathbf{C}_{z}\right) \text { (from (15) of Appendix A) } \\
& \leq \sum_{i=1}^{\alpha} \sum_{j=1}^{\beta} H\left(\mathbf{C}_{i, j}^{t} \mid \mathbf{C}_{i}\right) \text { (from (13) of Appendix A) } \\
& =0 \text { (from Property } 1 \text { of Definition 1). }
\end{aligned}
$$

The next lemma will be a useful tool to prove a lower bound on the size of the information distributed to servers from clients during their visits.

Lemma 5. Let $\mathcal{M}$ be an $\left(n, m, \tau, c, s,\left\{h_{j}\right\}_{j=1, \ldots, m}^{t=1, \ldots, \tau}\right)$ dynamic multi-threshold metering scheme. Let $\mathcal{S}_{1} \ldots, \mathcal{S}_{\beta}$ be a coalition of $\beta \leq s$ corrupt servers and let $B=\{1, \ldots, \beta\}$. Let $\mathcal{C}_{i}$ be a client and for $j=1, \ldots, \beta$ and $t=1, \ldots, \tau$, let $X_{j,\left(h_{j}^{t}-1\right)}^{t}$ be a set of visits from $h_{j}^{t}-1$ clients other than $\mathcal{C}_{i}$ to server $\mathcal{S}_{j}$ in time frame $t$. Then, for any $t=1, \ldots, \tau$ and $i=1, \ldots, n$, it holds that

$$
H\left(\mathbf{C}_{i, B}^{t} \mid \mathbf{X}_{1,\left(h_{1}^{t}-1\right)}^{t} \cdots \mathbf{X}_{\beta,\left(h_{\beta}^{t}-1\right)}^{t} \mathbf{S}_{B}^{t} \mathbf{V}_{B}^{[t-1]}\right) \geq H\left(\mathbf{P}_{B}^{t}\right) .
$$

Proof. Let $\mathcal{C}_{1}, \ldots, \mathcal{C}_{\alpha}$ be a coalition of $\alpha \leq c$ corrupt servers other than $\mathcal{C}_{i}$. Let us consider the random variables $\mathbf{E}=\mathbf{C}_{1} \ldots \mathbf{C}_{\alpha} \mathbf{X}_{1,\left(h_{1}^{t}-\alpha-1\right)}^{t} \ldots \mathbf{X}_{\beta,\left(h_{\beta}^{t}-\alpha-1\right)}^{t} \mathbf{S}_{B}^{t}$ $\mathbf{V}_{B}^{[t-1]}, \mathbf{A}=\mathbf{C}_{1, B}^{t} \ldots \mathbf{C}_{\alpha, B}^{t}, \mathbf{F}=\mathbf{C}_{i, B}^{t}$, and $\mathbf{G}=\mathbf{P}_{B}^{t}$. We have that

$$
\begin{aligned}
& H\left(\mathbf{C}_{1, B}^{t} \ldots \mathbf{C}_{\alpha, B}^{t} \mid \mathbf{C}_{1} \ldots \mathbf{C}_{\alpha} \mathbf{C}_{i, B}^{t} \mathbf{X}_{1,\left(h_{1}^{t}-\alpha-1\right)}^{t} \ldots \mathbf{X}_{\beta,\left(h_{\beta}^{t}-\alpha-1\right)}^{t} \mathbf{S}_{B}^{t} \mathbf{V}_{B}^{[t-1]}\right) \\
& \leq H\left(\mathbf{C}_{1, B}^{t} \ldots \mathbf{C}_{\alpha, B}^{t} \mid \mathbf{C}_{1} \ldots \mathbf{C}_{\alpha}\right) \text { (from (13) of Appendix A) } \\
&=0(\text { from Lemma 4). }
\end{aligned}
$$

Hence, A, E, and $\mathbf{F}$ verify the hypothesis of Lemma 2 and one has $H(\mathbf{G} \mid \mathbf{E F})=$ $H(\mathbf{G} \mid \mathbf{A E F})$, that is,

$$
\begin{aligned}
H( & \left.\mathbf{P}_{B}^{t} \mid \mathbf{C}_{1} \ldots \mathbf{C}_{\alpha} \mathbf{C}_{i, B}^{t} \mathbf{X}_{1,\left(h_{1}^{t}-\alpha-1\right)}^{t} \ldots \mathbf{X}_{\beta,\left(h_{\beta}^{t}-\alpha-1\right)}^{t} \mathbf{S}_{B}^{t} \mathbf{V}_{B}^{[t-1]}\right) \\
& =H\left(\mathbf{P}_{B}^{t} \mid \mathbf{C}_{1, B}^{t} \ldots \mathbf{C}_{\alpha, B}^{t} \mathbf{C}_{1} \ldots \mathbf{C}_{\alpha} \mathbf{C}_{i, B}^{t} \mathbf{X}_{1,\left(h_{1}^{t}-\alpha-1\right)}^{t} \ldots \mathbf{X}_{\beta,\left(h_{\beta}^{t}-\alpha-1\right)}^{t} \mathbf{S}_{B}^{t} \mathbf{V}_{B}^{[t-1]}\right) \\
& \leq H\left(\mathbf{P}_{B}^{t} \mid \mathbf{C}_{1, B}^{t} \ldots \mathbf{C}_{\alpha, B}^{t} \mathbf{C}_{i, B}^{t} \mathbf{X}_{1,\left(h_{1}^{t}-\alpha-1\right)}^{t} \ldots \mathbf{X}_{\beta,\left(h_{\beta}^{t}-\alpha-1\right)}^{t} \mathbf{S}_{B}^{t} \mathbf{V}_{B}^{[t-1]}\right) \\
& =H\left(\mathbf{P}_{B}^{t} \mid \mathbf{X}_{1,\left(h_{1}^{t}\right)}^{t} \ldots \mathbf{X}_{\beta,\left(h_{\beta}^{t}\right)}^{t} \mathbf{S}_{B}^{t} \mathbf{V}_{B}^{[t-1]}\right)
\end{aligned}
$$




$$
\begin{aligned}
& \leq \sum_{j=1}^{\beta} H\left(\mathbf{P}_{j}^{t} \mid \mathbf{X}_{j,\left(h_{j}^{t}\right)}^{t} \mathbf{S}_{j}^{t}\right)(\text { from } \underline{13}) \text { and }(15) \text { of Appendix A) } \\
& =0 \text { (from Property } 2 \text { of Definition 11). }
\end{aligned}
$$

From Property 3 of Definition 1 we have that

$$
H\left(\mathbf{P}_{B}^{t} \mid \mathbf{C}_{1} \ldots \mathbf{C}_{\alpha} \mathbf{X}_{1,\left(h_{1}^{t}-\alpha-1\right)}^{t} \ldots \mathbf{X}_{\beta,\left(h_{\beta}^{t}-\alpha-1\right)}^{t} \mathbf{S}_{B}^{t} \mathbf{V}_{B}^{[t-1]}\right)=H\left(\mathbf{P}_{B}^{t}\right) .
$$

Hence, $\mathbf{E}=\mathbf{C}_{1} \ldots \mathbf{C}_{\alpha} \mathbf{X}_{1,\left(h_{1}^{t}-\alpha-1\right)}^{t} \ldots \mathbf{X}_{\beta,\left(h_{\beta}^{t}-\alpha-1\right)}^{t} \mathbf{S}_{B}^{t} \mathbf{V}_{B}^{[t-1]}, \mathbf{F}=\mathbf{C}_{i, B}^{t}$, and $\mathbf{G}=$ $\mathbf{P}_{B}^{t}$ verify the hypothesis of Lemma 3 and one has $H(\mathbf{F} \mid \mathbf{E})=H(\mathbf{G})+H(\mathbf{F} \mid \mathbf{E G})$, that is

$$
\begin{aligned}
& H\left(\mathbf{C}_{i, B}^{t} \mid \mathbf{C}_{1} \ldots \mathbf{C}_{\alpha} \mathbf{X}_{1,\left(h_{1}^{t}-\alpha-1\right)}^{t} \ldots \mathbf{X}_{\beta,\left(h_{\beta}^{t}-\alpha-1\right)}^{t} \mathbf{S}_{B}^{t} \mathbf{V}_{B}^{[t-1]}\right) \\
& \quad=H\left(\mathbf{P}_{B}^{t}\right)+H\left(\mathbf{C}_{i, B}^{t} \mid \mathbf{C}_{1} \ldots \mathbf{C}_{\alpha} \mathbf{X}_{1,\left(h_{1}^{t}-\alpha-1\right)}^{t} \ldots \mathbf{X}_{\beta,\left(h_{\beta}^{t}-\alpha-1\right)}^{t} \mathbf{S}_{B}^{t} \mathbf{V}_{B}^{[t-1]} \mathbf{P}_{B}^{t}\right) \\
& \quad \geq H\left(\mathbf{P}_{B}^{t}\right) \text { (from (7) of Appendix A). }
\end{aligned}
$$

Moreover, $\mathbf{A}=\mathbf{C}_{1, B}^{t} \ldots \mathbf{C}_{\alpha, B}^{t}, \mathbf{E}=\mathbf{C}_{1} \ldots \mathbf{C}_{\alpha} \mathbf{X}_{1,\left(h_{1}^{t}-\alpha-1\right)}^{t} \ldots \mathbf{X}_{\beta,\left(h_{\beta}^{t}-\alpha-1\right)}^{t} \mathbf{S}_{B}^{t}$ $\mathbf{V}_{B}^{[t-1]}$, and $\mathbf{F}=\mathbf{C}_{i, B}^{t}$ verify the hypothesis of Lemma 2 and one has $H(\mathbf{F} \mid \mathbf{E})=$ $H(\mathbf{F} \mid \mathbf{A E})$, that is

$$
\begin{aligned}
H\left(\mathbf{C}_{i, B}^{t} \mid \mathbf{C}_{1} \ldots \mathbf{C}_{\alpha} \mathbf{X}_{1,\left(h_{1}^{t}-\alpha-1\right)}^{t} \ldots \mathbf{X}_{\beta,\left(h_{\beta}^{t}-\alpha-1\right)}^{t} \mathbf{S}_{B}^{t} \mathbf{V}_{B}^{[t-1]}\right) \\
\quad=H\left(\mathbf{C}_{i, B}^{t} \mid \mathbf{C}_{1, B}^{t} \ldots \mathbf{C}_{\alpha, B}^{t} \mathbf{C}_{1} \ldots \mathbf{C}_{\alpha} \mathbf{X}_{1,\left(h_{1}^{t}-\alpha-1\right)}^{t} \ldots \mathbf{X}_{\beta,\left(h_{\beta}^{t}-\alpha-1\right)}^{t} \mathbf{S}_{B}^{t} \mathbf{V}_{B}^{[t-1]}\right) \\
\leq H\left(\mathbf{C}_{i, B}^{t} \mid \mathbf{C}_{1, B}^{t} \ldots \mathbf{C}_{\alpha, B}^{t} \mathbf{X}_{1,\left(h_{1}^{t}-\alpha-1\right)}^{t} \ldots \mathbf{X}_{\beta,\left(h_{\beta}^{t}-\alpha-1\right)}^{t} \mathbf{S}_{B}^{t} \mathbf{V}_{B}^{[t-1]}\right) \\
\quad(\text { from (13) of Appendix A) } \\
=H\left(\mathbf{C}_{i, B}^{t} \mid \mathbf{X}_{1,\left(h_{1}^{t}-1\right)}^{t} \ldots \mathbf{X}_{\beta,\left(h_{\beta}^{t}-1\right)}^{t} \mathbf{S}_{B}^{t} \mathbf{V}_{B}^{[t-1]}\right)
\end{aligned}
$$

Therefore, the lemma follows from inequalities (3) and (21).

The next corollary immediately follows from Lemma 5. It implicitly shows that the size of the information each client has to give out when visiting a server is lower bounded by the size of the proof the server could reconstruct.

Corollary 6. Let $\mathcal{M}$ be an $\left(n, m, \tau, c, s,\left\{h_{j}\right\}_{j=1, \ldots, m}^{t=1, \ldots, \tau}\right)$ dynamic multi-threshold metering scheme. For any $i=1, \ldots, n, j=1, \ldots, m$, and $t=1, \ldots, \tau$, it holds that

$$
H\left(\mathbf{C}_{i, j}^{t}\right) \geq H\left(\mathbf{P}_{j}^{t}\right)
$$

If the proofs for the servers are uniformly chosen in a finite field $F$, that is $H\left(\mathbf{P}_{j}^{t}\right)=\log |F|$ for any $j=1, \ldots, m$ and $t=1, \ldots, \tau$, then from Corollary 6 and from (6) of Appendix A it holds that

$$
\log \left|C_{i, j}^{t}\right| \geq \log |F|
$$


for $i=1, \ldots, n, j=1, \ldots, m$, and $t=1, \ldots, \tau$. This bound is tight, as in Section 3 we have presented a protocol for an $\left(n, m, \tau, c, s,\left\{h_{j}\right\}_{j=1, \ldots, m}^{t=1, \ldots, \tau}\right)$ dynamic multi-threshold metering scheme in which the clients distribute exactly this information to servers during their visits.

In order to prove a lower bound on the size of the information distributed to clients we need the next lemma.

Lemma 7. Let $\mathcal{M}$ be an $\left(n, m, \tau, c, s,\left\{h_{j}\right\}_{j=1, \ldots, m}^{t=1, \ldots, \tau}\right)$ dynamic multi-threshold metering scheme. Let $\mathcal{S}_{1} \ldots, \mathcal{S}_{\beta}$ be a coalition of $\beta \leq s$ corrupt servers and let $B=\{1, \ldots, \beta\}$. Let $Z \subseteq\{1, \ldots, n\}$ be a set of client indices. Then, it holds that

$$
H\left(\mathbf{C}_{Z}\right) \geq \sum_{t=1}^{\tau} H\left(\mathbf{C}_{Z, B}^{t} \mid \mathbf{V}_{B}^{[t-1]}\right) .
$$

Proof. We have that

$$
\begin{aligned}
H\left(\mathbf{C}_{Z, B}^{1} \ldots \mathbf{C}_{Z, B}^{\tau} \mid \mathbf{C}_{Z}\right) & \leq \sum_{t=1}^{\tau} H\left(\mathbf{C}_{Z, B}^{t} \mid \mathbf{C}_{Z}\right)(\text { from (15) and (13) of Appendix A) } \\
& =0 \text { (from Lemma 4). }
\end{aligned}
$$

Therefore, applying Lemma 3 with $\mathbf{F}=\mathbf{C}_{Z, B}^{1} \ldots \mathbf{C}_{Z, B}^{\tau}$ and $\mathbf{D}=\mathbf{C}_{Z}$ we get

$$
\begin{aligned}
H\left(\mathbf{C}_{Z}\right) & =H\left(\mathbf{C}_{Z, B}^{1} \ldots \mathbf{C}_{Z, B}^{\tau}\right)+H\left(\mathbf{C}_{Z} \mid \mathbf{C}_{Z, B}^{1} \ldots \mathbf{C}_{Z, B}^{\tau}\right) \\
& \geq H\left(\mathbf{C}_{Z, B}^{1} \ldots \mathbf{C}_{Z, B}^{\tau}\right)(\text { from (17) of Appendix A) } \\
& =H\left(\mathbf{C}_{Z, B}^{1}\right)+\sum_{t=2}^{\tau} H\left(\mathbf{C}_{Z, B}^{t} \mid \mathbf{C}_{Z, B}^{1} \ldots \mathbf{C}_{Z, B}^{t-1}\right) \text { (from (14) of Appendix A) } \\
& \geq \sum_{t=1}^{\tau} H\left(\mathbf{C}_{Z, B}^{t} \mid \mathbf{V}_{B}^{[t-1]}\right) .
\end{aligned}
$$

The next lemma provides a lower bound on the size of the information distributed to clients during the initialization phase in dynamic multi-threshold metering schemes. It states that the information that must be kept secret by clients grows linearly with the number of time frames and the size of the coalition of corrupt servers.

Lemma 8. Let $\mathcal{M}$ be an $\left(n, m, \tau, c, s,\left\{h_{j}\right\}_{j=1, \ldots, m}^{t=1, \ldots, \tau}\right)$ dynamic multi-threshold metering scheme. Let $\mathcal{S}_{1} \ldots, \mathcal{S}_{\beta}$ be a coalition of $\beta \leq s$ corrupt servers and let $B=\{1, \ldots, \beta\}$. For any $i=1, \ldots, n$, it holds that

$$
H\left(\mathbf{C}_{i}\right) \geq \sum_{t=1}^{\tau} H\left(\mathbf{P}_{B}^{t}\right) .
$$

Proof. Let $\mathcal{C}_{i}$ be a client and for $j=1, \ldots, \beta$ and $t=1, \ldots, \tau$, let $X_{j,\left(h_{j}^{t}-1\right)}^{t}$ be a set of visits from $h_{j}^{t}-1$ clients other than $\mathcal{C}_{i}$ to server $\mathcal{S}_{j}$ in time frame $t$. We 
have that

$$
\begin{aligned}
H\left(\mathbf{C}_{i}\right) & \left.\geq \sum_{t=1}^{\tau} H\left(\mathbf{C}_{i, B}^{t} \mid \mathbf{V}_{B}^{[t-1]}\right) \text { (from Lemma } \mathbf{~}\right] \\
& \geq \sum_{t=1}^{\tau} H\left(\mathbf{C}_{i, B}^{t} \mid \mathbf{X}_{1,\left(h_{1}^{t}-1\right)}^{t} \ldots \mathbf{X}_{\beta,\left(h_{\beta}^{t}-1\right)}^{t} \mathbf{S}_{B}^{t} \mathbf{V}_{B}^{[t-1]}\right) \text { (from (13) of Appendix A) } \\
& \left.\geq \sum_{t=1}^{\tau} H\left(\mathbf{P}_{B}^{t}\right) \text { (from Lemma } 5\right)
\end{aligned}
$$

Notice that in Definition 1 we did not say anything on the entropies of random variables $\mathbf{P}_{j}^{t}$ for $j \in\{1, \ldots, m\}$ and $t \in\{1, \ldots, \tau\}$. Our results apply to the general case of arbitrary entropies on proofs, but for clarity, we state the next corollary for the simpler case that $H\left(\mathbf{P}_{j_{1}}^{t_{1}}\right)=H\left(\mathbf{P}_{j_{2}}^{t_{2}}\right)$ for all $j_{1}, j_{2} \in\{1, \ldots, m\}$ and $t_{1}, t_{2} \in\{1, \ldots, \tau\}$. We denote this common entropies by $H(\mathbf{P})$.

If the proof sequences of the corrupt servers are statistically independent, then the next corollary holds.

Corollary 9. Let $\mathcal{M}$ be an $\left(n, m, \tau, c, s,\left\{h_{j}\right\}_{j=1, \ldots, m}^{t=1, \ldots, \tau}\right)$ dynamic multi-threshold metering scheme and let $\mathcal{S}_{1}, \ldots, \mathcal{S}_{s}$ be the s corrupt servers. If the proof sequences of the $s$ corrupt servers are statistically independent, then it holds that

$$
H\left(\mathbf{C}_{i}\right) \geq s \tau H(\mathbf{P}),
$$

for any $i=1, \ldots, n$.

If the proofs for the servers are uniformly chosen in a finite field $F$, that is $H(\mathbf{P})=\log |F|$, then from Corollary 9 and from (6) of Appendix A it holds that

$$
\log \left|C_{i}\right| \geq s \tau \log |F|,
$$

for any $i=1, \ldots, n$. This bound is tight, as in Section 3 we have presented a protocol for an $\left(n, m, \tau, c, s,\left\{h_{j}\right\}_{j=1, \ldots, m}^{t=1, \ldots, \tau}\right)$ dynamic multi-threshold metering schemes which distributes exactly this information to clients.

\section{Efficiency of the Scheme}

In this section we analyze the efficiency of the scheme presented in Section 3 It is easy to see that the scheme meets the bounds (41) and (51) of Section 4. Indeed, during the initialization phase each client $\mathcal{C}_{i}$ receives by the audit agency the polynomial $Q(i, y)$, which is of degree $s \tau-1$. Therefore, the size of the information distributed to any client is $s \tau \log q$ and the bound (4) is tight. During a regular operation in a time frame $t$ each client $\mathcal{C}_{i}$ gives the value $Q(i, j \circ t)$ to the visited server $\mathcal{S}_{j}$. Therefore, the size of the information distributed to any visited server is $\log q$ and the bound (5) is tight. Hence, our protocol is optimal both with respect to the size of the information distributed to clients and with respect to the size of information given to servers by clients. This is important otherwise the task of receiving and sending information would burden the clients, that are not interested in the metering process. 


\section{Conclusions and Open Problems}

In this paper we have introduced dynamic multi-threshold metering schemes. In these schemes the servers need to communicate with the audit agency at the beginning of any time frame.

In this paper we have assumed that clients provide correct values when they visit servers. In a practical implementation of a metering scheme, some method of authentication should be used. However, the method of authentication used would be, in general, not dependent on the specific metering scheme and it could be incorporated as an additional feature, if desired.

We have proved lower bounds on the size of the information distributed to clients and on the size of the information given from clients to servers during their visits. An interesting problem would be to provide lower bounds on the size of the information distributed to servers at the beginning of any time frame and to devise dynamic multi-threshold metering schemes in which this information is as small as possible.

\section{Acknowledgements}

This work was done while the third author was visiting the Department of Combinatorics and Optimization at the University of Waterloo, Ontario, Canada. She wants to thank the Department for its hospitality. The research of the fourth author is supported by the Natural Sciences and Research Council of Canada under grants NSERC-IRC 216431-96 and NSERC-RGPIN 203114-98.

\section{A Information Theory Background}

In this Appendix we review the basic concepts of Information Theory used in our definitions and proofs. For a complete treatment of the subject the reader is advised to consult [2].

Given a probability distribution $\left\{\operatorname{Pr}_{\mathbf{x}}(x)\right\}_{x \in X}$ on a set $X$, we define the entropy 1 of $\mathbf{X}, H(\mathbf{X})$, as

$$
H(\mathbf{X})=-\sum_{x \in X} P r_{\mathbf{x}}(x) \log P r_{\mathbf{X}}(x)
$$

The entropy satisfies the following property

$$
0 \leq H(\mathbf{X}) \leq \log |X|
$$

where $H(\mathbf{X})=0$ if and only if there exists $x_{0} \in X$ such that $\operatorname{Pr}_{\mathbf{x}}\left(x_{0}\right)=1$; whereas, $H(\mathbf{X})=\log |X|$ if and only if $\operatorname{Pr}_{\mathbf{x}}(x)=1 /|X|$ for all $x \in X$.

\footnotetext{
${ }^{1}$ All logarithms in this paper are to the base 2 .
} 
Given two sets $X$ and $Y$ and a joint probability distribution on their cartesian product, the conditional entropy $H(\mathbf{X} \mid \mathbf{Y})$, is defined as

$$
H(\mathbf{X} \mid \mathbf{Y})=-\sum_{y \in Y} \sum_{x \in X} \operatorname{Pr}_{\mathbf{Y}}(y) \operatorname{Pr}(x \mid y) \log \operatorname{Pr}(x \mid y) .
$$

From the definition of conditional entropy it is easy to see that

$$
H(\mathbf{X} \mid \mathbf{Y}) \geq 0
$$

The mutual information $I(\mathbf{X} ; \mathbf{Y})$ between $\mathbf{X}$ and $\mathbf{Y}$ is defined by

$$
I(\mathbf{X} ; \mathbf{Y})=H(\mathbf{X})-H(\mathbf{X} \mid \mathbf{Y})
$$

and enjoys the following properties:

$$
I(\mathbf{X} ; \mathbf{Y})=I(\mathbf{Y} ; \mathbf{X})
$$

and $I(\mathbf{X} ; \mathbf{Y}) \geq 0$, from which one gets

$$
H(\mathbf{X}) \geq H(\mathbf{X} \mid \mathbf{Y})
$$

Given three sets $X, Y, Z$ and a joint probability distribution on their cartesian product, the conditional mutual information $I(\mathbf{X} ; \mathbf{Y} \mid \mathbf{Z})$ between $\mathbf{X}$ and $\mathbf{Y}$ given $\mathbf{Z}$ is

$$
I(\mathbf{X} ; \mathbf{Y} \mid \mathbf{Z})=H(\mathbf{X} \mid \mathbf{Z})-H(\mathbf{X} \mid \mathbf{Z} \mathbf{Y})
$$

and enjoys the following properties:

$$
I(\mathbf{X} ; \mathbf{Y} \mid \mathbf{Z})=I(\mathbf{Y} ; \mathbf{X} \mid \mathbf{Z})
$$

and $I(\mathbf{X} ; \mathbf{Y} \mid \mathbf{Z}) \geq 0$. Since the conditional mutual information is always non negative we get

$$
H(\mathbf{X} \mid \mathbf{Z}) \geq H(\mathbf{X} \mid \mathbf{Z Y})
$$

Given $n+1$ sets $X_{1}, \ldots, X_{n}, Y$ and a joint probability distribution on their cartesian product, the entropy of $\mathbf{X}_{1} \ldots \mathbf{X}_{n}$ given $\mathbf{Y}$ can be expressed as

$$
H\left(\mathbf{X}_{1} \ldots \mathbf{X}_{n} \mid \mathbf{Y}\right)=H\left(\mathbf{X}_{1} \mid \mathbf{Y}\right)+\sum_{i=2}^{n} H\left(\mathbf{X}_{i} \mid \mathbf{X}_{1} \ldots \mathbf{X}_{i-1} \mathbf{Y}\right)
$$

and enjoys the following property:

$$
H\left(\mathbf{X}_{1} \mathbf{X}_{2} \ldots \mathbf{X}_{n} \mid \mathbf{Y}\right) \leq \sum_{i=1}^{n} H\left(\mathbf{X}_{i} \mid \mathbf{Y}\right)
$$




\section{B Parameters and Variables Used in the Paper}

$\begin{array}{ll}n & \text { number of clients } \\ m & \text { number of servers } \\ \tau & \text { number of time frames } \\ c & \text { number of corrupt clients } \\ s & \text { number of corrupt servers } \\ h_{j}^{t} & \text { threshold for server } \mathcal{S}_{j} \text { in time frame } t \\ \mathbf{C}_{i} & \text { information distributed to client } \mathcal{C}_{i} \\ \mathbf{C}_{i, j}^{t} & \text { visit from client } \mathcal{C}_{i} \text { to server } \mathcal{S}_{j} \text { in time frame } t \\ B=\{1, \ldots, \beta\} & \text { indices of corrupt servers, } \beta \leq s \\ \mathbf{C}_{i, B}^{t} & \text { visits from client } \mathcal{C}_{i} \text { to servers } \mathcal{S}_{1}, \ldots, \mathcal{S}_{\beta} \text { in time frame } t \\ \mathbf{X}_{j,\left(d_{j}\right)}^{t} & \text { visits from } d_{j} \text { clients to server } \mathcal{S}_{j} \text { in time frame } t \\ \mathbf{S}_{j}^{t} & \text { information distributed to server } \mathcal{S}_{j} \text { at the beginning } \\ & \text { of time frame } t \\ \mathbf{S}_{B}^{t} & \text { information distributed to servers } \mathcal{S}_{1}, \ldots, \mathcal{S}_{\beta} \text { at the beginning } \\ & \text { of time frame } t \\ \mathbf{P}_{j}^{t} & \text { proof for server } \mathcal{S}_{j} \text { in time frame } t \\ \mathbf{P}_{B}^{t} & \text { proofs for servers } \mathcal{S}_{1}, \ldots, \mathcal{S}_{\beta} \text { in time frame } t \\ \mathbf{V}_{j}^{[t]} & \text { information collected by server } \mathcal{S}_{j} \text { in time frames } 1, \ldots, t \\ \mathbf{V}_{B}^{[t]} & \text { information collected by servers } \mathcal{S}_{1}, \ldots, \mathcal{S}_{\beta} \\ & \text { in time frames } 1, \ldots, t\end{array}$

\section{References}

1. C. Blundo, A. De Bonis and B. Masucci, Metering Schemes with Pricing, to appear in Proceedings of "14th International Symposium on DIStributed Computing DISC 2000", Toledo, Spain, October 4-6, 2000.

2. T. M. Cover and J. A. Thomas, Elements of Information Theory. John Wiley \& Sons, 1991.

3. A. De Bonis and B. Masucci, An Information Theoretical Approach to Metering Schemes, in Proceedings of "2000 International Symposium on Information Theory - ISIT 2000", Sorrento, Italy, June 25-30, 2000.

4. M. Franklin and D. Malkhi, Auditable Metering with Lightweight Security, in Proceedings of "Financial Cryptography '97", Lecture Notes in Computer Science, Vol. 1318, pp. 151-160, 1997.

5. B. Masucci and D. R. Stinson Efficient Metering Schemes with Pricing, submitted for publication, 2000. Technical Report CORR 2000-06, Centre for Applied Cryptographic Research, University of Waterloo.

6. B. Masucci and D. R. Stinson, Metering Schemes for General Access Structures, to appear in Proceedings of "6th European Symposium on Research in Computer Security - ESORICS 2000", Toulouse, France, October 4-6, 2000. Technical Report, CORR 2000-21, Centre for Applied Cryptographic Research, University of Waterloo. 
7. M. Naor and B. Pinkas, Secure and Efficient Metering, in Proceedings of "Advances in Cryptology - EUROCRYPT '98", Lecture Notes in Computer Science, Vol. 1403, pp. 576-590, 1998

8. M. Naor and B. Pinkas, Secure Accounting and Auditing on the Web, Computer Networks and ISDN Systems, Vol. 40, Issues 1-7, pp. 541-550, 1998.

9. A. Shamir, How to Share a Secret, Communications of the ACM, Vol. 22, pp. 612-613, 1979. 